05

\title{
Высокотемпературная сверхпроводниковая магнитная система для изучения нейронной активности
}

\author{
() Д.Н. Диев, И.А. Ковалев, М.Н. Макаренко, А.В. Наумов, А.В. Поляков, М.И. Сурин, \\ Д.И. Шутова, В.И. Щербаков
}

Национальный исследовательский центр „Курчатовский Институт“, 123182 Москва, Россия

e-mail: shutovadi@mail.ru, naumovandrej.isssph@yandex.ru

Поступило в Редакцию 18 мая 2021 г.

В окончательной редакции 18 ноября 2021 г.

Принято к публикации 19 ноября 2021 г.

\begin{abstract}
Описана сверхпроводниковая магнитная система (ВТСП СМС) для оснащения экспериментального исследовательского стенда по изучению экспрессии нейронов в постоянном и низкочастотном магнитном поле со значением индукции до 1 Т. Кратко перечислены особенности конструкции криомагнитной системы, ее электромагнитные и криогенные параметры. Приведены результаты сравнительных испытаний в жидком азоте при $77 \mathrm{~K}$ двух взаимозаменяемых криомагнитов. Первый магнит был изготовлен в виде двойной галеты из $4 \mathrm{~mm}$ высокотемпературной сверхпроводящей ленты второго поколения (ВТСП-2). Второй магнит выполнен из меди высокой чистоты по технологии бескаркасной намотки с пропиткой теплопроводящим эпоксидным клеем. Показано, что использование сверхпроводящего галетного магнита обладает рядом преимуществ по сравнению с криорезистивным соленоидом. Низкая энергоемкость ВТСП-2 установки позволит проводить длительное непрерывное in vitro наблюдение за биологическими объектами в магнитном поле.
\end{abstract}

Ключевые слова: сверхпроводимость, высокотемпературный сверхпроводник, ВТСП лента второго поколения, криомагнитная система, магнитное поле, нейронная активность.

DOI: $10.21883 /$ JTF.2022.02.52014.147-21

\section{Введение}

Техническая сверхпроводимость уже сегодня надежно служит медицине и биологии. Магниторезонансные томографы (МРТ), ядерно-магнитные резонансные (ЯМР) спектрометры и ускорители для протонной терапии онкологических заболеваний, изготовленные из низкотемпературных сверхпроводников (НТСП), сегодня стали незаменимыми инструментами в арсенале врачей и биологов $[1,2]$. При этом сверхпроводниковые технологии постоянно развиваются. Прогресс в области промышленного производства высокотемпературных сверхпроводящих лент второго поколения (ВТСП-2) [3] привел к появлению принципиально новой возможности конструирования экономичных сверхпроводящих магнитных систем (CMC) для генерации высоких магнитных полей при минимальных затратах на криогенное обеспечение.

Идет непрекращающийся научный поиск новых применений сверхпроводниковых технологий в генной инженерии и других медико-биологических исследованиях. Сверхпроводники незаменимы в создании высокополевых томографов нового поколения для наблюдения за мозговой активностью человека в процессе обработки информации. В качестве примера можно привести работы по созданию МРТ-магнита с индукцией магнитного поля в $11.7 \mathrm{~T}$ [4], а также проекты МРТ установок с магнитным полем в $20 \mathrm{~T}$ [5]. Другое перспективное направление - разработка метода доставки лекарствен- ных средств в конкретную область организма. В этой технологии положительно заряженные магнитные наночастицы, предварительно покрытые отрицательно заряженным биологически активным агентом, вводятся в живую ткань и направляются к очагу болезни с помощью локального источника внешнего магнитного поля. Исследования такого рода проводятся в офтальмологии, при лечении злокачественных образований и в генетических экспериментах [6,7]. Так, в работе [8] описан процесс разработки ВТСП-2 СМС, генерирующей магнитное поле с градиентом 20-70 T/m для изучения процессов генного транспорта. Также предлагаются проекты по замене постоянных магнитов в виде сборок Халбаха в биологических экспериментах на ВТСП-2 обмотки [9]. В магнитной энцефалографии (МЭГ) сверхпроводящие квантовые интерференционные датчики (СКВИДы) регистрируют чрезвычайно малые (примерно $10^{-15} \mathrm{~T}$ ) электромагнитные сигналы мозга, а ВТСП-2 ленты используются для магнитного экранирования [10]. Настоящая работа связана с еще одной нишей применения СМС в биологических исследованиях, а именно с созданием устройств для изучения нейронной активности под действием магнитного поля.

Выяснение механизмов работы мозга является интереснейшей фундаментальной задачей медицинской физики. Ее решение может привести к открытию эффективных методов лечения целого ряда заболеваний, а также к разработке принципиально новых вычислительных 
устройств и систем управления, имитирующих работу мозга.

Оптогенетический метод является одним из способов исследования нервной деятельности. В этом методе в мембраны нервных клеток вводятся специальные светочувствительные каналы (опсины), и активация или деактивация нейронов происходит под действием света с определенной длиной волны. Очевидным недостатком данного метода является его инвазивность - требуется удаление части черепа и вживление в мозг оптических волокон.

Альтернативой может стать магнитогенетическая технология для неинвазивной стимуляции определенных когнитивных функций мозга (например, специфических следов памяти). В этом методе под воздействием магнитного поля происходит избирательная активация отдельной популяции нейронных сетей мозга, помеченных магниточувствительными белками. Явное преимущество магнитного поля, используемого в качестве активатора, заключается в большей (по сравнению со светом) глубине проникновения в живые ткани. Максимальная измеренная глубина проникновения магнитного поля в структуры мозга $-9 \mathrm{~cm}$.

Большая часть магнитогенетических экспериментов [11-14] проводится в два этапа. Сначала нейроны подвергаются воздействию магнитного поля, затем происходит наблюдение за поведением объекта исследования. С биологической же точки зрения намного интереснее непрерывно наблюдать за изменением поведения нейронов, находящихся в постоянном или переменном магнитном поле.

Считается, что наиболее вероятно получить нейронный отклик при трех видах магнитного воздействия: при продолжительном нахождении объекта исследования в постоянном магнитном поле с индукцией до $1 \mathrm{~T}$ с непрерывным мониторингом его состояния, при воздействии переменного поля с частотой до $100 \mathrm{~Hz}$ и амплитудой до $0.5 \mathrm{~T}$, а также при воздействии переменного поля с частотой до $500 \mathrm{kHz}$ и амплитудой до $50 \mathrm{mT}$.

Выбор источника магнитного поля для магнитогенетических экспериментов является оптимизационной задачей, зависящей от особенностей конкретных опытов. Стоимость изготовления магнитной системы и эксплуатационные расходы также имеют не последнее значение. Самым доступным источником магнитного поля являются постоянные магниты на основе редкоземельных элементов с игольчатыми концентраторами магнитного потока, которые наиболее часто используются в нейронных исследованиях. Однако их возможности ограничены получением постоянного магнитного поля с индукцией менее $0.5 \mathrm{~T}$ на образце. Резистивные неохлаждаемые электромагниты обладают неприемлемо высоким уровнем электропотребления.

Решением могут стать криомагнитные системы. Медные обмотки, охлаждаемые жидким азотом, благодаря своей простоте могут использоваться в непродолжительных экспериментальных сессиях для генерации перемен- ных магнитных полей интенсивностью до 0.5 Т. ВСТП-2 $\mathrm{CMC}$, несмотря на относительно сложную технологию изготовления обмоток и высокую стоимость проводника, являются универсальными источниками магнитного поля. При температуре $65-77 \mathrm{~K}$ в сочетании с концентраторами магнитного потока они способны генерировать как постоянное, так и переменное магнитное поле с амплитудой $1 \mathrm{~T}$ и выше в течение длительного времени при малом расходе электроэнергии [15].

С 2019г. мы работаем над созданием криомагнитной системы для специализированного экспериментального стенда лаборатории механизмов и технологий памяти отдела нейронаук НБИКС-центра НИЦ „Курчатовский институт" по изучению экспрессии нейронов in vitro в постоянном и низкочастотном (до $100 \mathrm{~Hz}$ ) магнитном поле [16-18]. Необходимость размещения криостата с СМС на уже существующем стенде на базе исследовательского микроскопа Nikon Eclipse TI, изначально предназначенного для оптогенетических исследований, накладывает ряд ограничений на конструкцию криомагнитной системы. Кроме того, на сегодняшний день пока еще нет четкой уверенности в том, какой рабочий режим магнита будет самым востребованным. Поэтому в рамках НИОКР мы изготовили и испытали два взаимозаменяемых магнита, рассчитанных на работу при $T=65-77 \mathrm{~K}$ : криорезистивный из меди высокой чистоты и сверхпроводящий из ВТСП-2 лент.

В следующих разделах приведены особенности конструкции криомагнитной системы, ее электрические и криогенные параметры, технология изготовления двух магнитов, а также результаты их испытаний в жидком азоте.

\section{1. Криомагнитная система}

Эскизы криомагнитной системы для нейронных исследований и фотография изготовленного криостата представлены на рис. 1 и 2. Магнит размещается в криостате с жидким азотом. Контейнер $D=36 \mathrm{~mm}$ с биологическим объектом исследования располагается на $30 \mathrm{~mm}$ ниже нижнего торца магнита. Для концентрации магнитного потока на образце используется цилиндрический сердечник из пермендюра 49К2Ф диаметром $30 \mathrm{~mm}$, размещенный в несквозном „теплом“ отверстии криостата. Нижний торец сердечника прижат к крышке контейнера для образцов. Диагностика криомагнитной системы включает в себя три температурных датчика и два датчика Холла для измерения магнитного поля.

Испаряющийся азот проходит через латунные токовводы с развитой охлаждаемой поверхностью. При проведении длительных биологических экспериментов заливочная горловина криостата может служить терминалом для подключения криогенного питателя (LN2 feeder) - рис. 2, a. Последний представляет собой сосуд Дьюара с запасом хладагента и патрубками для его подачи под собственным весом в основной криостат. 


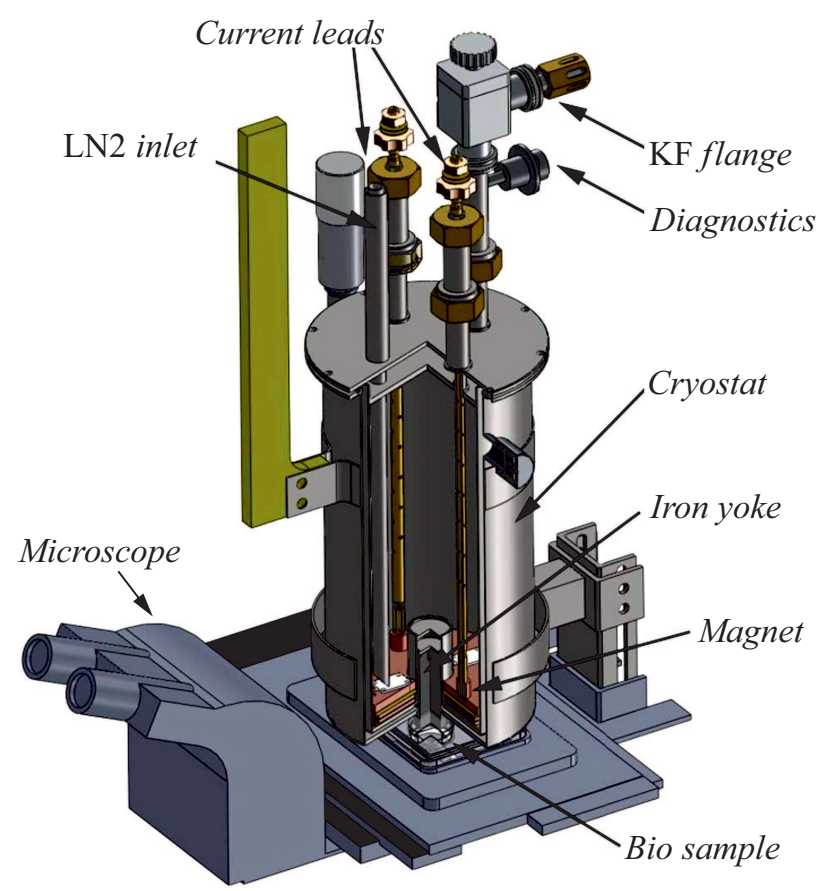

Рис. 1. Трехмерный эскиз высокотемпературной сверхпроводящей магнитной системы для нейронных исследований в сборке с микроскопом.

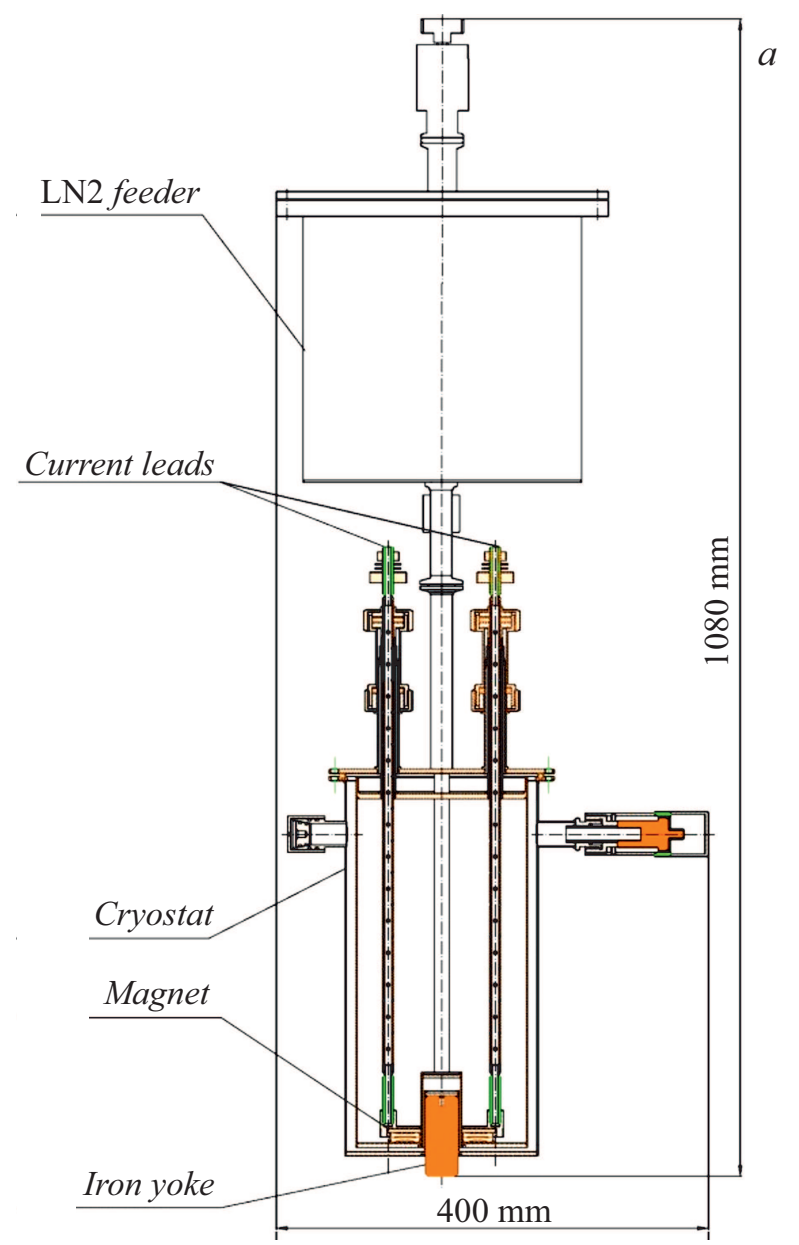

Конструкция криостата предусматривает возможность работы в переохлажденном азоте под откачкой при $T=65 \mathrm{~K}$ с целью повышения рабочего тока ВТСП-2 магнита и создаваемой им величины магнитной индукции. Этот метод уже был опробован нами ранее [19]. В настоящий момент все детали криостата изготовлены и находятся в процессе сборки (рис. 2,b).

\section{2. Магниты}

Взаимозаменяемые магниты из медного провода и ВТСП-2 ленты показаны на рис. 3 и 4. Сравнение их основных рабочих характеристик приведено в таблице. Бескаркасный медный магнит, изготовленный в виде многослойного соленоида, при намотке пропитывался трехкомпонентным криогенным эпоксидным клеем. Для улучшения теплопередачи с внутренних витков криорезистивной обмотки в хладагент использован порошок оксида алюминия $\left(\mathrm{Al}_{2} \mathrm{O}_{3}\right)$, теплопроводность которого при температуре $77-40 \mathrm{~K}$ в 2-6 раз выше, чем у меди [20]. На 2 весовых доли клея приходилась 1 весовая доля добавки.

Сверхпроводящий магнит, намотанный из $4 \mathrm{~mm}$ BТСП-2 ленты производства компании SuperOx в ла-

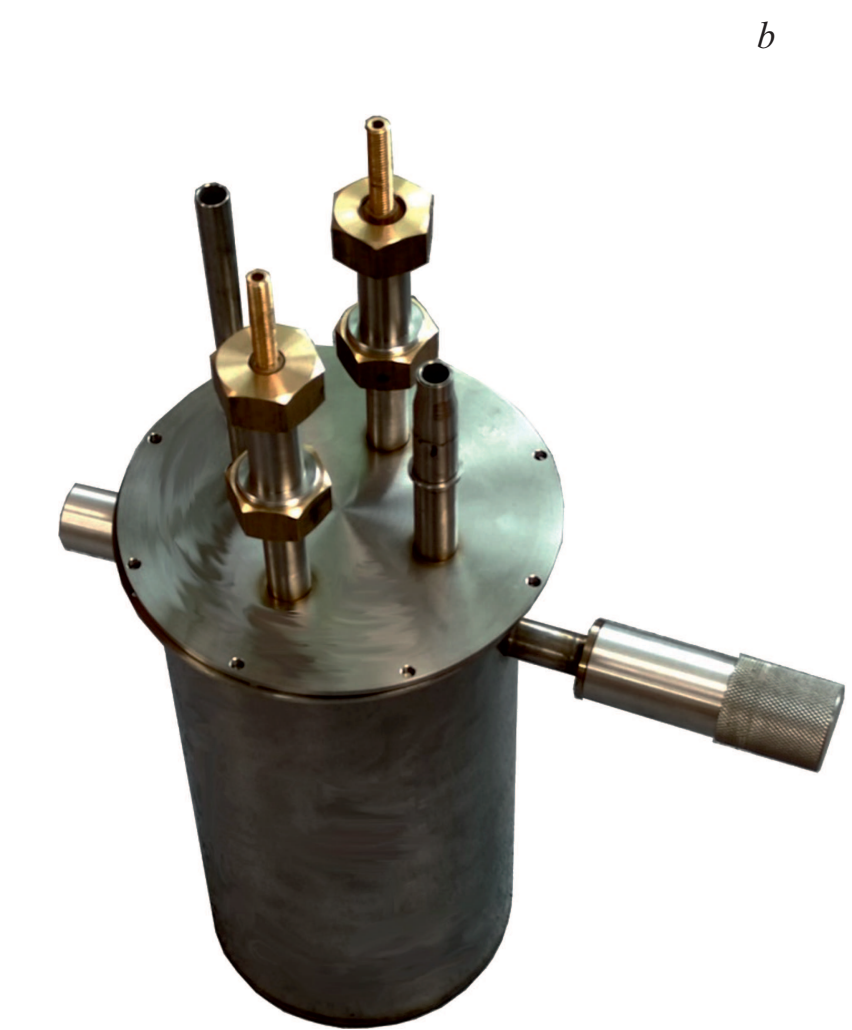

Рис. 2. Габаритные размеры высокотемпературной сверхпроводящей магнитной системы $(a)$ и ее криостат в сборе $(b)$. 


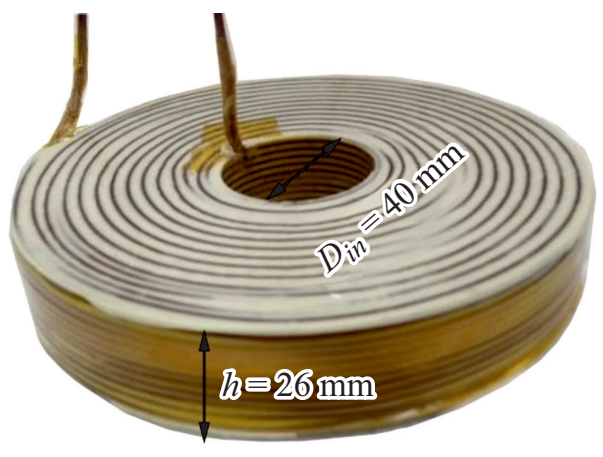

Рис. 3. Резистивный магнит после намотки.
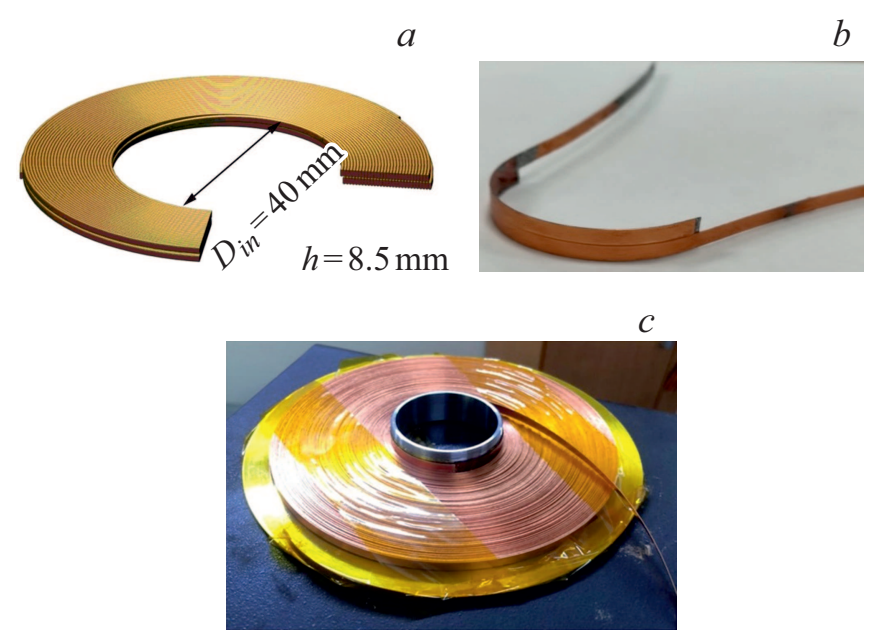

Рис. 4. Модель двойной ВТСП-2 галеты с размерами (a); спай ВТСП-2 лент на внутреннем витке галеты $(b)$, двойная ВТСП-2 галета на стальном каркасе в процессе намотки $(c)$.

ковой полиимидной изоляции, имеет вид встречной двойной галеты с внутренним спаем. Секции галеты изолированы друг от друга полиимидной прокладкой. Внутренний спай ВТСП-2 галеты (рис. 4,b) был изготовлен при помощи вспомогательного формера, диаметр которого совпадал с внутренним диаметром каркаса галеты. Предварительно измеренное в жидком азоте сопротивление ВТСП спая оказалось равно $116 \mathrm{n} \Omega$ при $77 \mathrm{~K}$.

Расчет распределения индукции магнитного поля в области расположения биологического образца для обоих магнитов с сердечниками был выполнен в пакете Finite Element Method Magnetics (FEMM) [21]. Программа позволяет моделировать осесимметричные электромагнитные поля методом конечных элементов и может использоваться для решения электромагнитных двумерных задач. Геометрия магнитной системы, заложенная в расчет, показана на рис. 5. Расчетная плотность тока в обмотках задана в таблице.

Результаты расчетов для сверхпроводящего и резистивного магнитов в сборке с концентратором магнитного потока из пермендюра 49К2Ф представлены на рис. 6. Видно, что оба магнита в сборе с теплым сердечником способны генерировать магнитное поле с величиной индукции $\sim 0.5 \mathrm{~T}$ при температуре жидкого

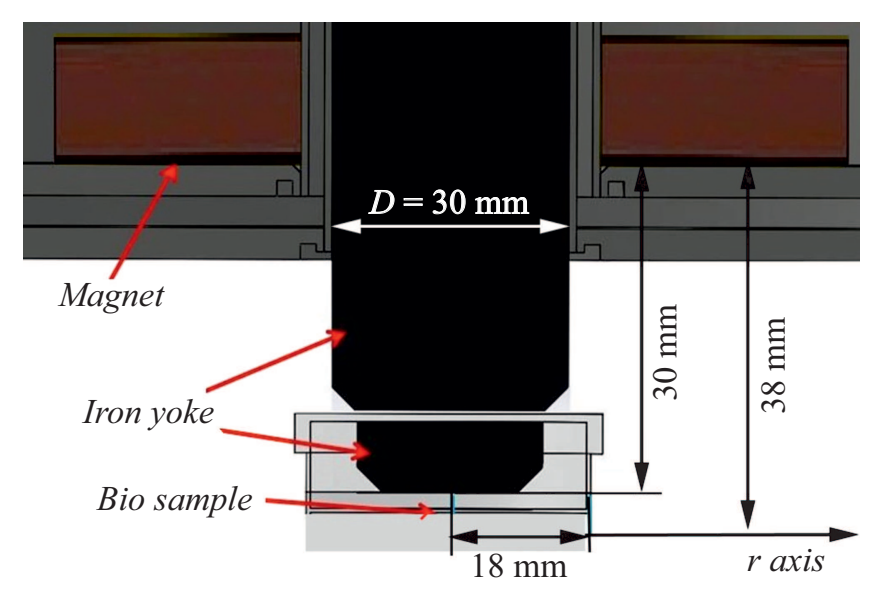

Рис. 5. Схема магнитной системы для расчета распределения индукции магнитного поля на биологическом образце.
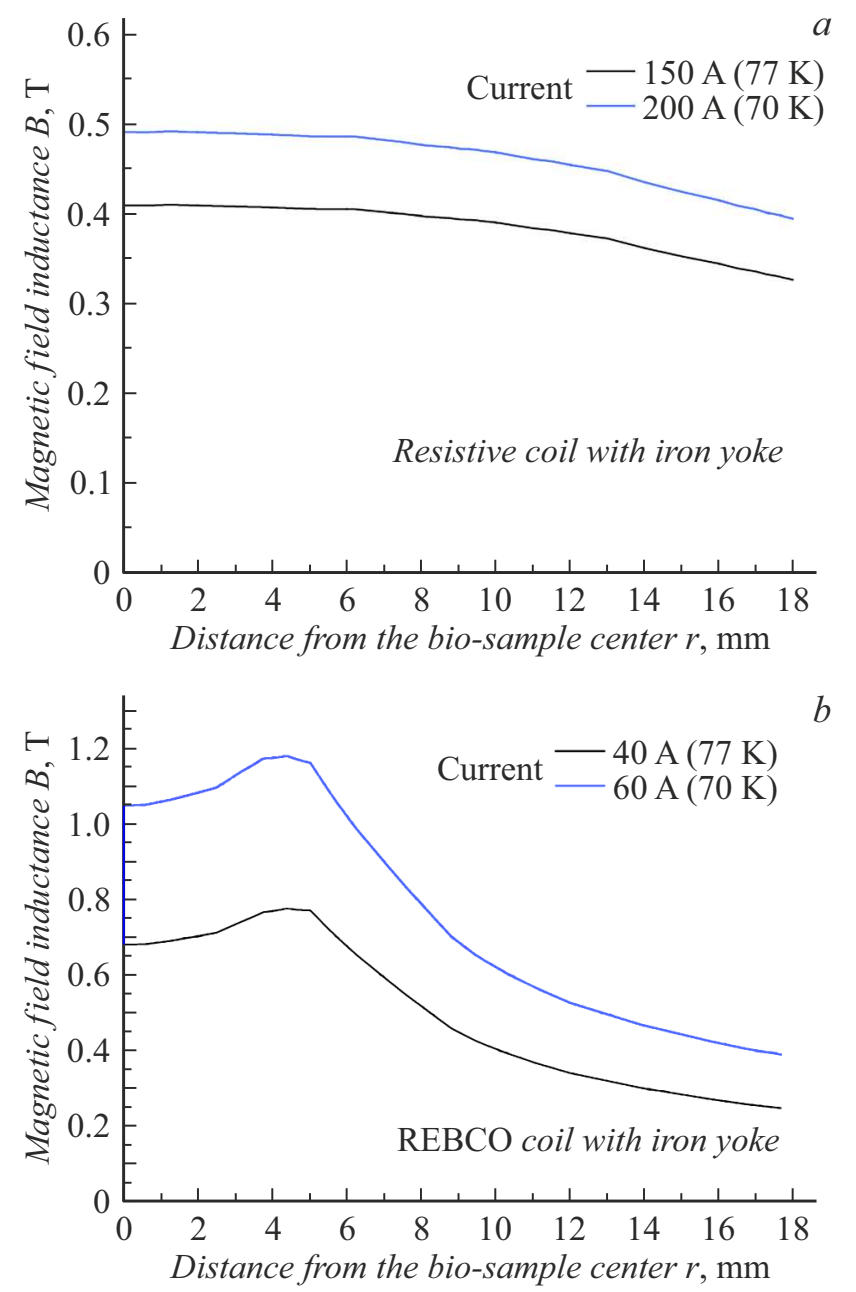

Рис. 6. Расчетное распределение индукции магнитного поля на биологическом образце: резистивный магнит с сердечником $(a)$, ВТСП-2 магнит с сердечником $(b)$. 
Характеристики криомагнитов

\begin{tabular}{|c|c|c|}
\hline Вид магнита & Сверхпроводящий & Резистивный \\
\hline Проводник & $\begin{array}{l}\text { ВТСП-2 лента } \\
4 \times 0.1 \mathrm{~mm}, \text { SuperOx, } \\
\text { Ic_min }(77 \mathrm{~K})=110 \mathrm{~A}\end{array}$ & $\begin{array}{l}\text { Провод круглого } \\
\text { сечения из меди М0, } \\
D=2.1 \mathrm{~mm}\end{array}$ \\
\hline Вид изоляции & $\begin{array}{l}\text { Каптоновый лак с толщиной } \\
\text { покрытия в } 10 \mu \mathrm{m}\end{array}$ & $\begin{array}{l}\text { Лак МЛ-92 с толщиной } \\
\text { покрытия } 50 \mu \mathrm{m}\end{array}$ \\
\hline Сопротивление магнита, $\Omega$ & $116 \cdot 10^{-9}(77 \mathrm{~K})-$ спай & $\begin{array}{l}0.06(77 \mathrm{~K}) \\
0.052(70 \mathrm{~K}) \\
0.047(65 \mathrm{~K})\end{array}$ \\
\hline Форма обмотки & $\begin{array}{l}\text { Двойная встречная галета } \\
\text { Сухая намотка без пропитки }\end{array}$ & $\begin{array}{l}\text { Многослойный соленоид, } \\
\text { замоноличенный эпоксидной } \\
\text { смолой ЭД- } 20 \text { с добавкой } \mathrm{Al}_{2} \mathrm{O}_{3} \\
\text { в весовом соотношении } 2: 1\end{array}$ \\
\hline Материал каркаса & $12 \mathrm{X} 18 \mathrm{H} 10 \mathrm{~T}$ & Без каркаса \\
\hline Диаметр внутренний, mm & 40 & 40 \\
\hline Диаметр наружный, mm & 135 & 140 \\
\hline Высота, mm & 8.5 & 26 \\
\hline Число витков & $\begin{array}{l}236 \text { на каждую секцию галеты, } \\
\text { итого } 472 \text { витка }\end{array}$ & 299 \\
\hline Плотность намотки, $1 / \mathrm{cm}^{2}$ & 117 & 23 \\
\hline Рабочий ток при $77 \mathrm{~K}, \mathrm{~A}$ & 40 & 150 \\
\hline $\begin{array}{l}\text { Рабочая плотность тока } \\
\text { при } 77 \mathrm{~K}, \mathrm{~A} / \mathrm{mm}^{2}\end{array}$ & 47 & 35 \\
\hline Длина проводника, m & 130 & 93 \\
\hline $\begin{array}{l}\text { Экспериментальный коэффи- } \\
\text { циент поле/ток, mT/A }\end{array}$ & 7.2 & 4.13 \\
\hline Индуктивность, mH & 18 & 4 \\
\hline $\begin{array}{l}\text { Тепловыделение в обмотке } \\
\text { на постоянном рабочем токе, W }\end{array}$ & $<1(65-77 \mathrm{~K})$ & $\begin{array}{l}1350(77 \mathrm{~K}) \\
1170(70 \mathrm{~K}) \\
1060(65 \mathrm{~K})\end{array}$ \\
\hline
\end{tabular}

азота $(T=77 \mathrm{~K})$. При работе с переохлажденным жидким азотом под откачкой $(T=65-70 \mathrm{~K})$ ВТСП-2 магнит с сердечником позволит получить поле выше $1 \mathrm{~T}$.

Также из таблицы и расчетных кривых на рис. 6 хорошо видны преимущества ВТСП-2 магнита по сравнению с медным. Главный недостаток резистивного магнита высокое энергопотребление. При токе (150-200) А и $T=77 \mathrm{~K}$ оно составит $1.35-2.40 \mathrm{~kW}$, что соответствует расходу жидкого азота в 30-541/h. По этой причине криорезистивный соленоид может быть использован в краткосрочных биологических экспериментах с постоянным полем, а также для генерации переменного магнитного поля с частотой до $100 \mathrm{~Hz}$.

Благодаря почти вдвое более высокому коэффициенту поле/ток, ВТСП-2 магнит вводит сердечник в зону магнитного насыщения. С одной стороны, это позволяет получить максимальное значение магнитной индукции на образце $B_{\max }=1.2 \mathrm{~T}$ (при работе магнита в переохлажденном азоте), с другой - приводит к большей неоднородности индукции магнитного поля на образце (рис. 6, $b$ ).

Криорезистивный соленоид не насыщает сердечник, оставляя связь между магнитным полем и током в соленоиде линейной, а поле на образце - более однородным. Однако максимальное значение индукции магнитного поля в этом случае не превышает $B_{\max }=0.5 \mathrm{~T}$ (рис. 6,a).

Оптимальные параметры магнитных полей (частота, амплитуда, однородность) для магнитогенетических исследований пока не известны. Все исследования такого рода носят поисковый характер, поэтому на данной стадии предсказать, как повышенная неоднородность 
$a$

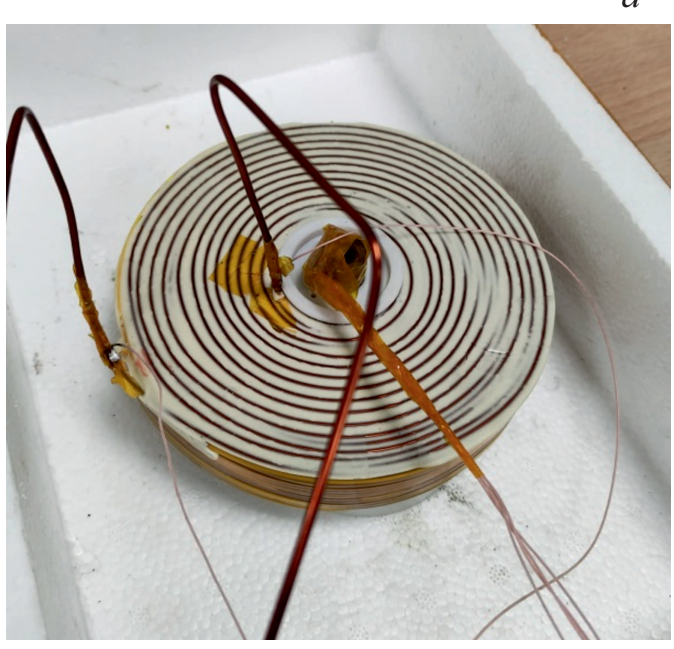

$c$

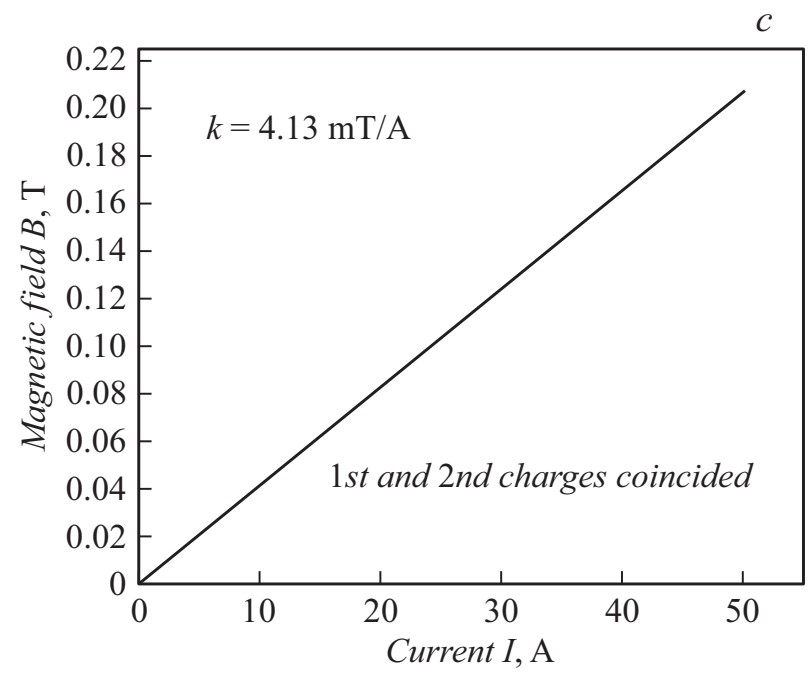

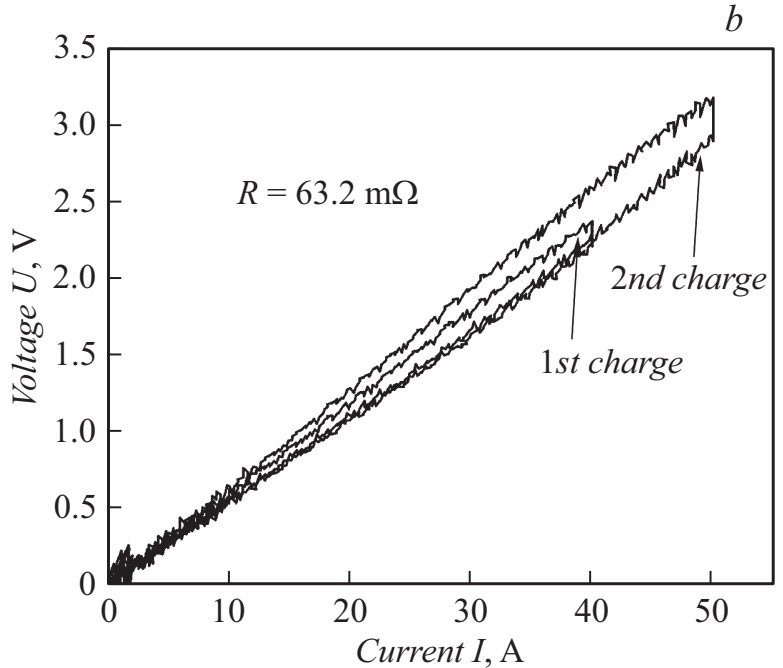

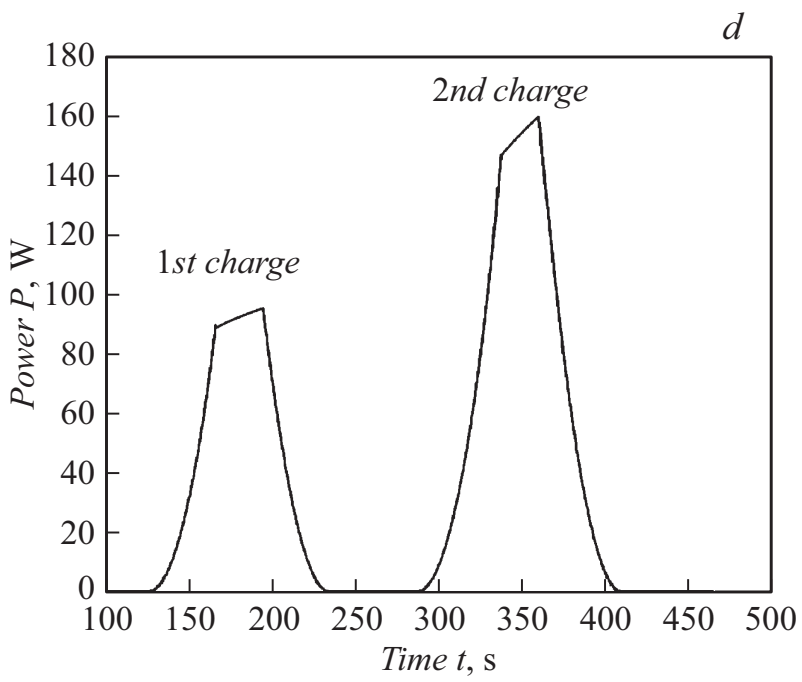

Рис. 7. Резистивный соленоид в процессе проведения испытаний $(a)$; вольт-амперная характеристика резистивного соленоида (два ввода) $(b)$; зависимость величины магнитной индукции в центре резистивного магнита от тока $(c)$; зависимость мощности, выделяющейся в резистивном магните от времени $(d)$.

внешнего магнитного поля в случае ВТСП-2 магнита повлияет на биологические образцы, нельзя. Возможно, два изготовленных нами взаимозаменяемых криомагнита с различной однородностью магнитного поля позволят провести сравнительные исследования такого рода после установки магнитной системы на микроскоп.

\section{3. Испытания магнитов}

Предварительные испытания двух магнитов проводились поочередно в открытой азотной ванне без пермендюрового сердечника. Ввод тока осуществлялся программируемым источником тока Lambda GEN10-500. Скорость ввода тока $0.2 \mathrm{~A} / \mathrm{s}$. Для защиты источника при выводе тока параллельно тестируемому магниту подключался защитный диод.

Сигналы с токоизмерительного шунта, датчика Холла и напряжения на соленоиде регистрировались много- канальной измерительной системой NI CompactDAQ. Датчики Холла располагались в геометрическом центре магнитов. Процесс и результаты испытаний бескаркасного резистивного соленоида представлены на рис. 7. Ток вводился в два этапа: первый ввод - до $40 \mathrm{~A}$, затем вывод до нуля, и затем второй ввод до 50 А. Измеренный коэффициент поле/ток (4.13 mT/A) совпадает с расчетным значением (4.39 mT/A), которое было получено методом конечных элементов, с погрешностью $5.8 \%$.

Из рис. 7, $b, d$ виден разогрев резистивной обмотки при протекании тока. Другими словами, тепловыделение в магните под нагрузкой превышало возможности теплосъема жидкого азота, даже несмотря на присутствие теплопроводящей добавки в эпоксидном клее, которым были пропитаны витки магнита. Из проведенных опытов можно сделать вывод, что работать с резистивным криомагнитом возможно в коротких испытательных сессиях. 


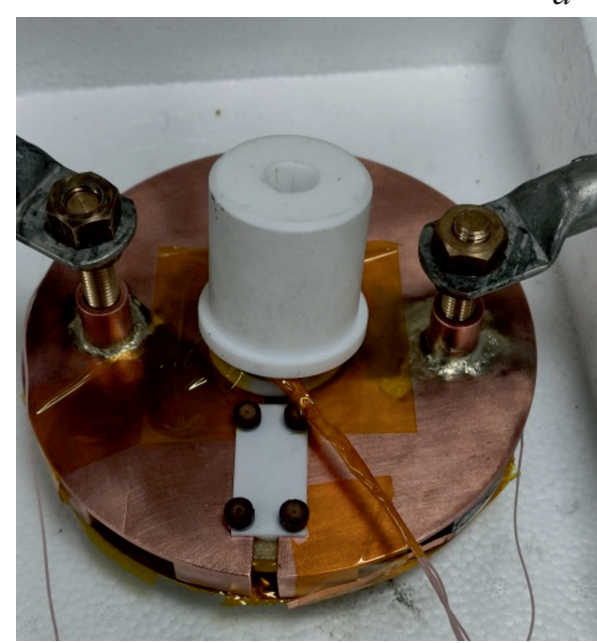

$c$

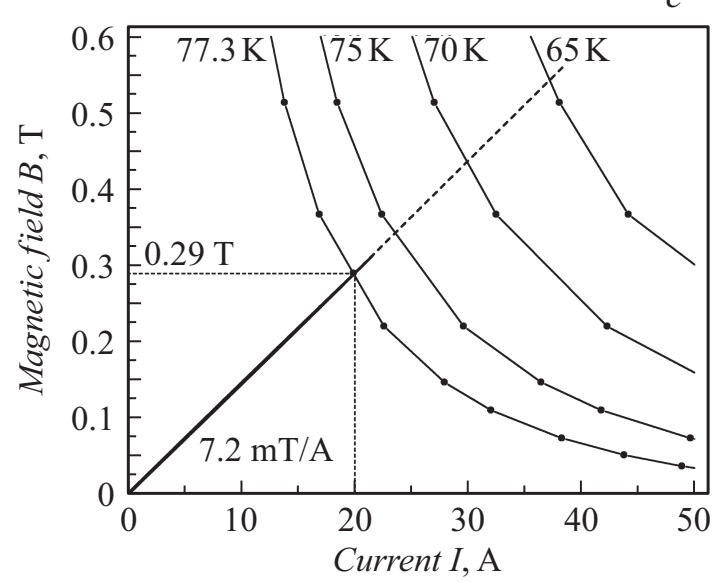

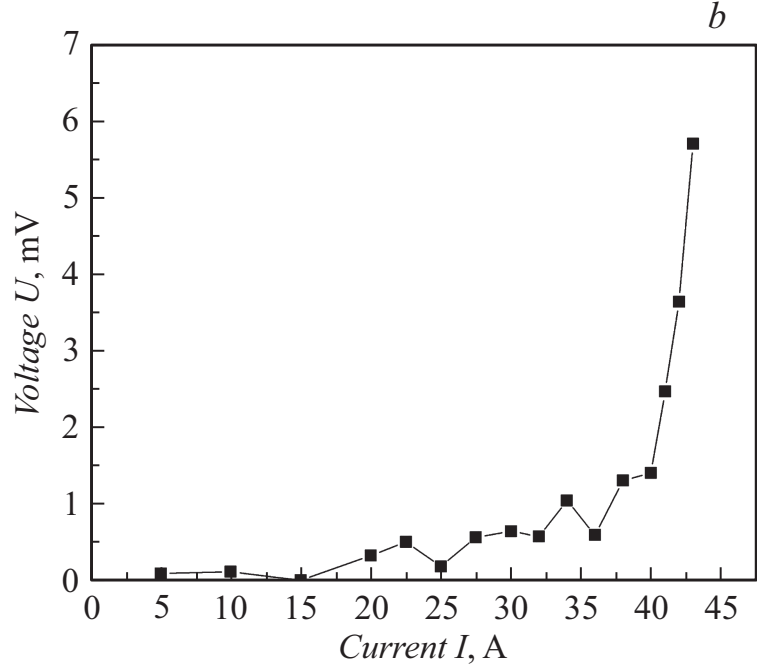

$b$

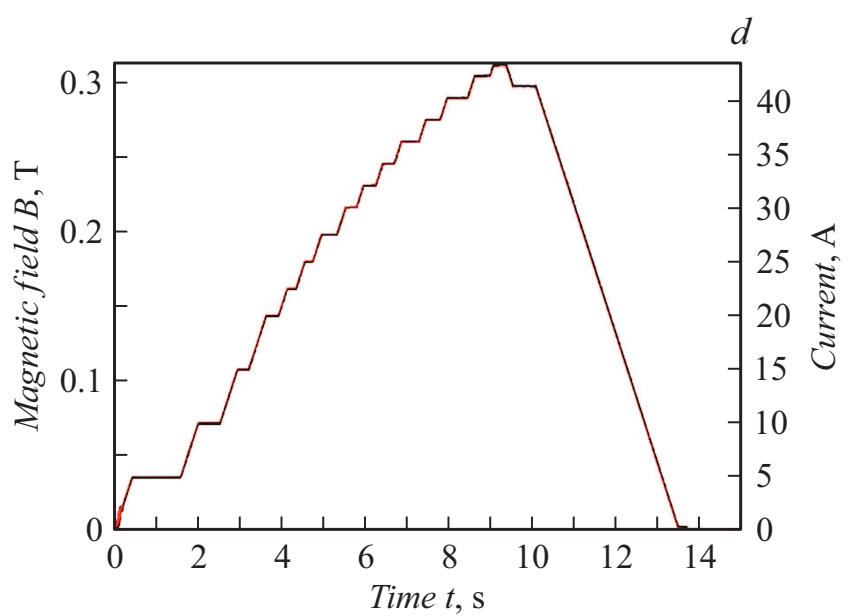

Рис. 8. ВТСП-2 магнит в процессе проведения испытаний $(a)$; вольт-амперная характеристика ВТСП-2 магнита $(b)$; нагрузочная кривая ВТСП-2 магнита без сердечника $(c)$; зависимость величины тока и магнитной индукции в центре ВТСП-2 магнита без сердечника от времени $(d)$.

Результаты испытаний ВТСП-2 магнита приведены на рис. 8. Для компенсации реактивной составляющей напряжения при испытаниях сверхпроводящего магнита ввод тока производился ступенчато. Критический ток ВТСП-2 галеты, определенный по критерию $0.1 \mu \mathrm{V} / \mathrm{cm}$, оказался равен $\sim 42$ А при температуре $77 \mathrm{~K}$, что соответствует индукции магнитного поля в центре магнита $B=0.3$ Т и хорошо согласуется с расчетной нагрузочной кривой ВТСП-2 магнита (рис. $8, c$ ). Экспериментальный коэффициент поле/ток оказался равен $7.20 \mathrm{mT} / \mathrm{A}$, что совпадает с расчетной величиной $7.53 \mathrm{mT} / \mathrm{A}$, полученной методом конечных элементов, в пределах 4.0\% погрешности. Генерация тепла в ВТСП-2 магните обусловлена только наличием спая и не превышает $1 \mathrm{~W}$.

\section{Выводы}

Исследование поведения нейронов в магнитном поле может привести лучшему пониманию механизмов мышления и формирования памяти, открытию новых способов лечения ряда заболеваний, а также к созданию алгоритмов для вычислительных машин нового поколения.

Применение сверхпроводниковых магнитных систем открывает новые возможности для биологических исследований. Оснащение исследовательского биологического стенда на базе микроскопа Nikon Eclipse TI в лаборатории Биомедицинского центра НИЦ „Курчатовский институт“ сверхпроводниковой магнитной системой позволит проводить длительное непрерывное in vitro наблюдение за биологическими объектами в магнитном поле с индукцией до 1 T.

Результаты предварительных сравнительных испытаний в жидком азоте показали явное преимущество ВТСП-2 магнита по сравнению с криорезистивным на постоянном токе. В качестве следующих шагов мы планируем испытания изготовленных криомагнитов на переменном токе с частотами до $100 \mathrm{~Hz}$ в рабочем криостате стенда в сборе с пермендюровым сердечни- 
ком, а также финальную установку СМС на стенде по изучению поведения нейронов в магнитном поле в связке с микроскопом. Дальнейшее развитие данной темы предполагает создание опытной ВТСП-2 криомагнитной системы для размещения животных в магнитном поле и исследования когнитивных процессов и функций памяти in vivo. В случае успеха открывается перспектива создания принципиально новой неинвазивной технологии специфической магнитогенетической стимуляции когнитивных сетей головного мозга, которую можно будет использовать для лечения заболеваний центральной нервной системы, скрининга фармпрепаратов, управления биопротезами и т.д.

\section{Благодарности}

Авторы выражают благодарности: Соловьеву Д.Ю., Лазареву И.Ю., Ильинскому А.А., Оленеву А.О. за активное участие в изготовлении магнитов и подготовке к экспериментам.

\section{Финансирование работы}

Работа выполнена при финансовой поддержке Национального исследовательского центра „Курчатовский Институт“, приказ № 1055 от 02.07.2020.

\section{Конфликт интересов}

Авторы заявляют, что у них нет конфликта интересов.

\section{Список литературы}

[1] J. Minervini, M. Parizh, M. Schippers. Supercond. Sci. Technol., 31, 030301 (2018). https://doi.org/10.1088/1361-6668/aaa826

[2] А.В. Максимов, В.В. Кирьянова, М.А. Максимова. Физиотерапия, бальнеология и реабилитация, 3, 34 (2013).

[3] A. Molodyk, S. Samoilenkov, A. Markelov, P. Degtyarenko, S. Lee, V. Petrykin, M. Gaifullin, A. Mankevich, A. Vavilov, B. Sorbom, J. Cheng, S. Garberg, L. Kesler, Z. Hartwig, S. Gavrilkin, A. Tsvetkov, T. Okada, S. Awaji, D. Abraimov, A. Francis, G. Bradford, D. Larbalestier, C. Senatore, M. Bonura, A.E. Pantoja, S.C. Wimbush, N.M. Strickland, A. Vasiliev. Sci. Rep., 11, 2084 (2021). DOI: $10.1038 / \mathrm{s} 41598-021-81559-\mathrm{z}$

[4] D. Le Bihan, T. Schild. Supercond. Sci. Technol., 30 (3), 033003 (2017). DOI: 10.1088/1361-6668/30/3/033003

[5] T.F. Budinger, M.D. Bird, L. Frydman, J.R. Long, Th.H. Mareci, W.D. Rooney, B. Rosen, J.F. Schenck, V.D. Schepkin, A.D. Sherry, D.K. Sodickson, Ch.S. Springer, K.R. Thulborn, K. Uğurbil, L.L. Wald. Magn. Reson. Mater. Phys., Biol. Med., 29 (3), 617 (2016). DOI: $10.1007 / \mathrm{s} 10334-016-0561-4$

[6] D. Zahn, K. Klein, P. Radon, D. Berkov, S. Erokhin, E. Nagel, M. Eichhorn, F. Wiekhorst, S. Dutz. Nanotechnol., 31, 95101 (2020). DOI: DOI: $10.1088 / 1361-6528 / a b b 0 b 4$

[7] D. Li, Y. Ren. IEEE Trans. Appl. Supercond., 28 (6), 1 (2018). DOI: $10.1109 /$ tasc.2018.2836999
[8] T. Hasegawa, H. Nakagam, Y. Akiyama, S. Nishijima. Prog. Supercond. Cryog., 19 (1), 9 (2017). DOI: $10.9714 /$ psac.2017.19.1.009

[9] B. Shen, J. Geng, C. Li, X. Zhang, L. Fu, H. Zhang, Jun Ma, T.A. Coombs. Physica C, 538, 46 (2017). DOI: $10.1016 /$ j.physc.2017.05.009

[10] K. Narasaki, S. Tsunematsu. IOP Conf. Series, 755, 012107 (2020). DOI: 10.1088/1757-899X/755/1/012107

[11] G. Wang, P. Zhang, S.K. Mendu, Y. Wang, Ya. Zhang, Xi Kang, B.N. Desai, J.J. Zhu. bioRxiv preprint (2019). http://dx.doi.org/10.1101/737254

[12] M.A. Wheeler, C.J. Smith, M. Ottolini, B.S. Barker, A.M. Purohit, R.M. Grippo, R.P. Gaykema, A.J. Spano, M.P. Beenhakker, S. Kucenas, M.K. Patel, Ch.D. Deppmann, A.D. Güler. Nat. Neurosci., 19 (5), 756 (2016). DOI: $10.1038 / \mathrm{nn} .4265$

[13] S.A. Stanley, L. Kelly, K.N. Latcha, S.F. Schmidt, X. Yu1, A.R. Nectow, J. Sauer, J.P. Dyke, J.S. Dordick, J.M. Friedman. Nature, 531, 647 (2016). DOI: 10.1038/nature17183

[14] S.A. Stanley, J. Sauer, R.S. Kane, J.S. Dordick, J.M. Friedman. Nature Medicine, 21 (1), 92 (2015). DOI: 10.1038/nm.3730

[15] A.V. Polyakov, V.I. Shcherbakov, S.A. Shevchenko, M.I. Surin. Phys. Procedia, 45, 241 (2013). DOI: $10.1016 /$ j.phpro.2013.05.012

[16] A.V. Naumov, I.A. Kovalev, D.N. Diev, A.O. Olenev, A.V. Polyakov, M.I. Surin, V.I. Shcherbakov. Nanotech. in Russia, 14, 613 (2019). DOI: 10.1134/S1995078019060119

[17] Ресурсные центры Курчатовского комплекса НБИКС природоподобных технологий, OnLine: http://www.rc.nrcki.ru/pages/main/neuron/index.shtml

[18] Д.Н. Диев, И.А. Ковалев, М.Н. Макаренко, А.В. Наумов, А.В. Поляков, М.И. Сурин, Д.И. Шутова, В.И. Щербаков. Электричество, 8, 13 (2021). DOI: $10.24160 / 0013-5380-2021-8-13-20$

[19] D.N. Diev, M.N. Makarenko, A.V. Naumov, A.V. Polyakov, V.I. Shcherbakov, D.I. Shutova, M.I. Surin. Prog. Supercond. Cryog., 21 (3), 47 (2019). DOI: 10.9714/psac.2019.21.3.047

[20] Я.Л. Абелиов. ВИАМ/2005-204279, Клеи. Герметики. Технологии, 8, (2005).

[21] Электронный ресурс. Режим доступа: FEMM https://www.femm.info/wiki/Download 